\title{
EFICIENCIA DE TRAMPAS “PITLIGHT” CON LED PARA EL MUESTREO DE COLEOPTERA NOCTURNOS (INSECTA) EN SELVAS TROPICALES
}

\section{EFFICIENCY OF PITLIGHT TRAPS WITH LED FOR SAMPLING NOCTURNAL COLEOPTERA (INSECTA) IN TROPICAL FORESTS}

\author{
Cisteil X. PÉREZ-HERNÁndeZ, ${ }^{1, *}$ Martha Isabel LUNA-GÓMEZ, ${ }^{1}$ Aldo Eric \\ FUENTES BARRADAS, ${ }^{1}$ LUIS ALBERTO RODRÍGUEZ MIRANDA, ${ }^{1}$ DARIANA R. GUERRERO \\ FUENTES, ${ }^{1}$ MIREYA RAMÍREZ BALLESTEROS, ${ }^{1}$ FABIOLA GARCÍA CALZADA, ${ }^{1}$ ÁNGEL \\ RODRÍGUEZ-MORENO ${ }^{1}$ Y GABRIEL GUTIÉRREZ-GRANADOS ${ }^{1,2}$
}

\begin{abstract}
${ }^{1}$ Departamento de Zoología, Instituto de Biología, Universidad Nacional Autónoma de México. Apartado postal 70-153, 04510 México, Ciudad de México, México.

${ }^{2}$ Facultad de Estudios Superiores Zaragoza, UNAM. Batalla 5 de mayo esq, Fuerte de Loreto, Col. Ejército de Oriente, C.P. 09230, Iztapalapa, CDMX, México <moxybelis25@gmail.com>, <aldoeric.fuentes@gmail.com>, <betoskalaria@hotmail.com>,<darguerrero03@gmail.com>, <mballesteros@ciencias.unam.mx>, <fgc220987@gmail.com>, <tanicandil@hotmail.com>, <gabgg@st.ib.unam.mx>.

* Autora de correspondencia: <cxinum@gmail.com>
\end{abstract}

Recibido: 02/09/2016; aceptado: 31/05/2017

Editor responsable: Cuauhtémoc Deloya.

Pérez-Hernández, C. X., Luna-Gómez, M. I., Fuentes Barradas, A. E., Rodríguez Miranda, L. A., Guerrero Fuentes, D. R., Ramírez Ballesteros, M., García Calzada, F., Rodríguez-Moreno, A. y Gutiérrez-Granados, G. (2017). Eficiencia de trampas “pitlight” con LED para el muestreo de Coleoptera nocturnos (Insecta) en selvas tropicales. Acta Zoológica Mexicana (n.s.) 33(2), 314327.

RESUMEN. Se evaluó el uso de trampas de caída con luz portables ("pitlight”) con diodos emisores de luz (LED) para la captura de Coleoptera nocturnos en una selva tropical, con la finalidad de proveer herramientas eficientes para el monitoreo de insectos. Se comparó la diversidad de familias y morfoespecies recolectada con trampas de caída pasivas ("pitfall”) y con trampas de caída con luz ("pitlight”) de tres distintas longitudes de onda (azul, blanca y amarilla), bajo la hipótesis de que las trampas pitlight son más eficientes, y que los insectos serán más atraídos por las longitudes de onda más cortas y de alta frecuencia. Además, el muestreo se dividió en dos períodos nocturnos de cinco horas, con el objetivo de analizar el tiempo necesario para obtener una colecta significativa. Las recolectas con trampas pitlight fueron notablemente superiores a las pitfall pasivas, y aunque no se encontraron diferencias significativas entre los valores de riqueza, abundancia y diversidad de coleópteros obtenidos con tres colores de LED (azul, blanco, amarillo), ni entre los horarios nocturnos de recolecta, sí se observó una alta disimilitud en la composición de morfoespecies entre los distintos tratamientos. Las trampas pitlight con LED son un método sencillo, portátil y económico que es capaz de obtener una captura eficiente y representativa en periodos de tiempo muy cortos, por lo que se recomienda su uso para el inventario de comunidades de insectos. Palabras clave: Coleoptera, diodos emisores de luz, bosque tropical perennifolio.
Pérez-Hernández, C. X., Luna-Gómez, M. I., Fuentes Barradas, A. E., Rodríguez Miranda, L. A., Guerrero Fuentes, D. R., Ramírez Ballesteros, M., García Calzada, F., RodríguezMoreno, A., \& Gutiérrez-Granados, G. (2017). Efficiency of pitlight traps with LED for sampling nocturnal Coleoptera (Insecta) in tropical forests. Acta Zoológica Mexicana (n.s.) 33(2), 314-327.

ABSTRACT. In this study, with the aim to provide more efficient tools for insect monitoring, we test the efficiency of pitlight traps with light-emitting diodes (LED) in captures of nocturnal Coleoptera from a tropical forest. Under the hypothesis that pitlights are more efficient and that insects would be mainly attracted by shorter and higher frequency wavelengths, families and morphospecies diversity obtained with passive pitfall and pitlight traps of three different colors (blue, white and yellow light) was compared. In addition, with the purpose to analyze the necessary time to obtain a significant sample of Coleoptera, sampling was carried out in two nocturnal periods of five hours. Captures with pitlight traps were noticeably superior to that with passive pitfalls and, even though there were no significant differences between richness, abundance and diversity values obtained for three color of LED (blue, white, yellow), not even among both nocturnal sampling periods, we found a high dissimilarity in morphospecies composition among them. Pitlight traps with LED are a simple, portable and inexpensive method able to obtain an efficient and significant capture in short time periods, so we recommend their use for the inventory of Coleoptera communities.

Key words: Sampling, light-emitting diodes, tropical rain forest. 


\section{INTRODUCCIÓN}

Los Coleoptera (Insecta) conforman un taxón hiperdiverso que comprende más de 392,400 especies (Zhang, 2013). Estos artrópodos han colonizado numerosos hábitats y se consideran importantes indicadores ecológicos que dependen de factores complejos, como puede ser la estructura de la vegetación. Debido a esto, el monitoreo de su riqueza y abundancia provee información útil acerca del estado de los ecosistemas y las comunidades que habitan (Alexander, 2014), por lo que es necesario implementar métodos adecuados para la obtención de datos de campo sobre este grupo (Fagundes et al., 2011).

Uno de los métodos frecuentemente utilizados para la colecta de coleópteros y de otros invertebrados son las trampas de caída pasivas ("pitfall”), principalmente por su simplicidad, bajo costo y poco esfuerzo invertido por el colector (Hansen \& New, 2005; Holopainen, 1992; Woodcock, 2014). Sin embargo, debido a la constante necesidad de obtener información de manera rápida acerca de taxones muy diversos, la propuesta continua de métodos de colecta que permitan tener una representación significativa de la biodiversidad en corto tiempo es de gran utilidad (Alonso et al., 2011).

Una desventaja importante de las trampas pitfall pasivas frente a otros métodos de colecta, como la red de golpeo, las trampas de intercepción, trampas con cebo e inclusive la recolecta manual, es que la cantidad de ejemplares que se obtienen es mucho menor (Cutz-Pool \& Virginia, 2013), debido a que su captura es incidental, es decir, la caída de los organismos en las trampas es azarosa. Por ello, el diseño e implementación de las trampas pitfall está en constante cambio, en respuesta a la continua búsqueda de mayor eficiencia en la captura de organismos. Por otro lado, la eficiencia del muestreo de insectos con este tipo de trampas, también depende de variaciones en el diseño de las trampas, el tamaño del recipiente, la adición de barreras, embudos o a la implementación de cebos (como tejido en descomposición, necrotrampas) o fuentes de luz (trampas pitlight; Hansen \& New, 2005).

De manera particular, la adición de una fuente luminosa a las trampas pitfall, ha resultado ser muy eficiente para el muestreo de insectos (Heap, 1988; pitlight Luminoc ${ }^{\circledR}$, Hébert et al., 2000). Y la implementación de trampas modificadas de esta manera para la recolecta de coleópteros, ha mostrado mayor efectividad en la captura de individuos, especies y familias; incluso ha permitido la obtención de especies raras de familias como Byrrhidae, Melandryidae, Scraptiidae, Stenotrachelidae y Throsci- dae, lo que destaca la importancia de este método para la caracterización precisa de comunidades de coleópteros nocturnos (Hébert et al., 2000).

Si bien los modelos de pitlight propuestos anteriormente son muy eficientes (Heap, 1988; Hébert et al., 2000), tienen la desventaja de que requieren de una fuente de energía poderosa (batería tipo linterna de $6 \mathrm{~V}$, aproximadamente $600 \mathrm{gr}, 68 \mathrm{~mm} \times 68 \mathrm{~mm} \times 115 \mathrm{~mm}$ ) para mantener encendido un tubo fluorescente por largos periodos de tiempo (112 horas; 4 horas/noche en 28 días) (Biocom, 2014). Este problema se elimina fácilmente mediante el uso de diodos emisores de luz, mejor conocidos como LED (por sus siglas en inglés), que pueden ser alimentados con una batería de $9 \mathrm{~V}$, de menor costo económico (aproximadamente MX\$85 vs MX\$420), de menor tamaño y peso que las de $6 \mathrm{~V}$ (aproximadamente $46 \mathrm{gr}$, 48,5 $\mathrm{mm} \times 26,5 \mathrm{~mm} \times 17,5 \mathrm{~mm}$ ) y que puede funcionar entre 60 y 120 horas, dependiendo del número de LED que se utilice. Los LED son una tecnología desarrollada para producir un sistema de iluminación más eficaz, menos costoso y con mayor tiempo de vida útil que los sistemas incandescentes y fluorescentes (Cohnstaedt et al., 2008; Price \& Baker, 2016), pero ha sido poco utilizada en la colecta de organismos nocturnos con fototropismo positivo, como muchas especies de escarabajos (e.g. Price \& Baker, 2016). La implementación de esta fuente de luz en otro tipo de trampas para capturar insectos ha tenido resultados prometedores y ha mostrado diferencias significativas en la composición de especies capturadas con distintos colores de LED (e.g. Diptera, Burkett et al., 1998). En dípteros, también se ha observado que las trampas que utilizan LED como fuente lumínica, han aumentado hasta 50\% la tasa de captura al tiempo que reducen el consumo de energía entre $50 \%$ y $60 \%$, en comparación con las trampas luminosas que emplean focos incandescentes y que emiten una mayor cantidad de luz en el espectro infrarrojo (Cohnstaedt et al., 2008).

Diversos experimentos han evaluado la respuesta de los insectos a la luz como un cebo y, aunque se ha demostrado que algunas especies presentan fototropismo negativo (Alcázar-Ruiz et al., 2003; Méndez-Aguilar et al., 2005), numerosos artículos han comprobado que muchos artrópodos presentan fototropismo positivo, y que responden de acuerdo con la longitud de onda de luz empleada (Chagnon et al., 2000; Heap, 1988; Therrien et al., 1999); se considera que los artrópodos son atraídos principalmente a longitudes de onda corta y de alta frecuencia, debido a que las detectan más fácilmente (Ashfaq et al., 2005). 
Aparte, algunos insectos nocturnos tienen mayores picos de actividad al anochecer, respecto a los que la presentan al amanecer (Racey \& Swift, 1985). Tomando lo anterior en consideración, se recomienda restringir el muestreo con trampas de atracción luminosa a un solo periodo de la noche, para maximizar el esfuerzo de colecta (e.g. Scalercio et al., 2009). Si el muestreo se diseña de esta manera, el consumo de energía es menor y la recolecta puede concentrarse en los periodos con mayor probabilidad de obtener la mayor diversidad de especies.

Considerando las recomendaciones anteriores y basándonos en la hipótesis de que los Coleoptera nocturnos tienen mayor atracción por longitudes de onda corta y de alta frecuencia, en este estudio presentamos una evaluación rápida de la fauna de coleópteros de un solo sitio. Para ello se modificó el modelo de trampa pitlight propuesto por Heap (1988), mediante la adición de LED en tres colores (blanco, amarillo y azul) que emiten luz en distintas longitudes de onda. El principal objetivo del estudio fue contestar las siguientes preguntas: en términos de abundancia, riqueza y diversidad de especies 1) ¿ Son más eficientes las trampas pitlight con LED que las trampas pitfall pasivas, 2) ¿ Existen diferencias en la eficiencia de captura entre diferentes longitudes de onda de luz (diferentes colores de LED)?, y 3) ¿Existe variación en la composición de especies capturadas en distintos períodos durante la noche?, en este caso, de 19:30 a 00:30 horas y de 01:00 a 06:00 horas.

\section{MATERIALES Y MÉTODOS}

Área de estudio. El estudio se llevó a cabo en la Estación de Biología Tropical "Los Tuxtlas" del Instituto de Biología de la Universidad Nacional Autónoma de México (UNAM), ubicada dentro de la Reserva de la Biosfera Los Tuxtlas. La estación se localiza entre los $95^{\circ} 04^{\prime}$ y $95^{\circ} 09$ longitud Oeste y los $18^{\circ} 34^{\prime}$ y $18^{\circ} 36^{\prime}$ de latitud Norte, en el municipio de San Andrés Tuxtla, Veracruz, México. El tipo de vegetación predominante es selva alta perennifolia, con clima cálido-húmedo, temperatura media anual de $25^{\circ} \mathrm{C}$ y precipitación anual de $4500 \mathrm{~mm}$ (Conanp, 2006). Dentro de los terrenos de la estación se eligió un sitio homogéneo, en términos de vegetación y pendiente; el área total de muestreo fue de 2,400 $\mathrm{m}^{2}$.

Diseño de trampas pitfall y pitlight. Cada trampa de caída consistió de un recipiente de plástico de un litro de capacidad, con un techo circular fijo y separado $15 \mathrm{~cm}$ de la boca del recipiente (Fig. 1). En el caso de las trampas pitlight, al interior del techo se añadió una tira flexible - dispuesta en forma circular- de seis LED (modelo 3528) de uno de tres colores distintos: 1) LED azules, que emiten luz en una longitud de onda de 430 a $505 \mathrm{~nm}$ y están hechos de nitruro de galio; 2) LED amarillos, con una longitud de onda de $585 \mathrm{~nm}$ a $595 \mathrm{~nm}$ y están elaborados con fosfito de aluminio indio galio y un tinte de carburo de silicona; y 3) LED blancos, que emiten luz en una longitud de onda de $470 \mathrm{~nm}$ y están construidos con un tinte de carburo de silicona (Drenen et al., 2000). La

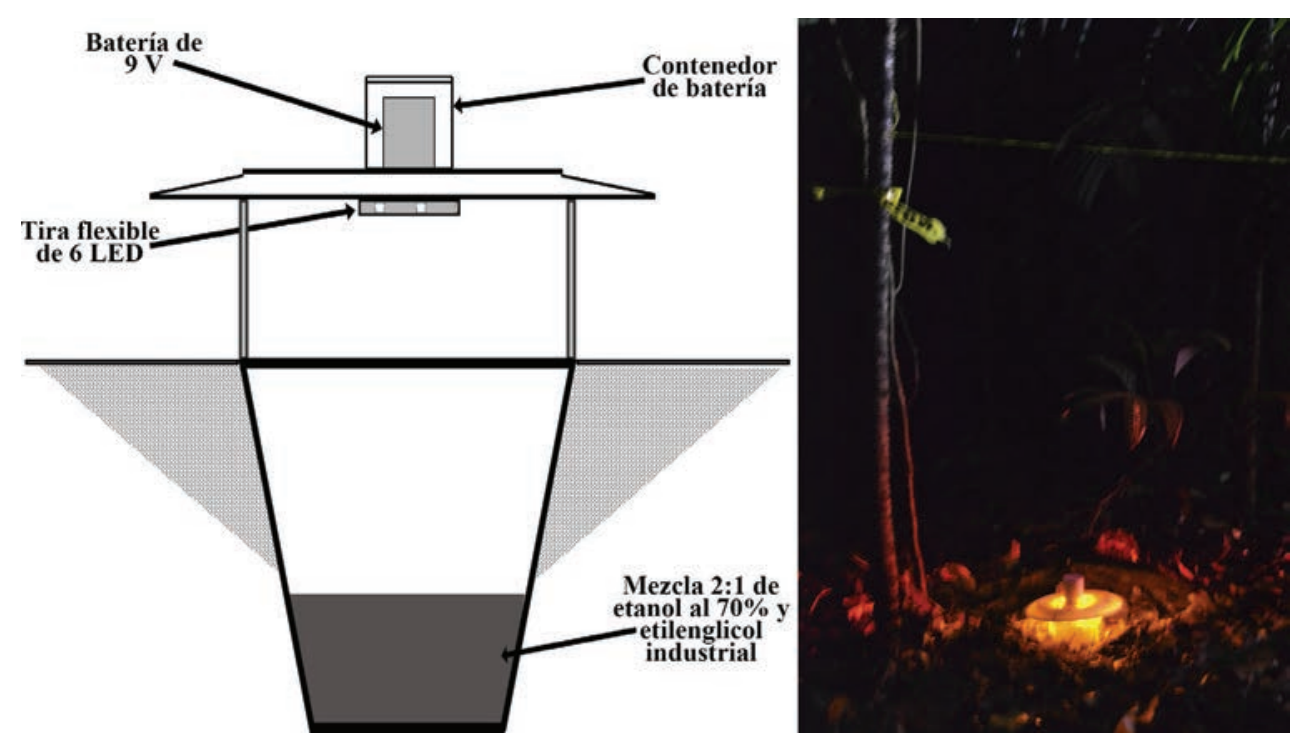

Figura 1. Diseño de la trampa “pitlight” con LED utilizada en el presente estudio. 
tira de LED se alimentaba de una batería de $9 \mathrm{~V}$ marca Duracell ${ }^{\circledR}$ a través de un cable de conexión, que estaba colocada en la parte exterior del techo de la trampa. A cada trampa pitfall o pitlight se añadió una mezcla 2:1 de etanol al 70\% y anticongelante (etilenglicol industrial) hasta un tercio de su capacidad, como conservador y para disminuir la evaporación, respectivamente.

Obtención de datos. Se trazaron cuatro transectos de 80 $\mathrm{m}$ de largo, paralelos y separados entre sí por $10 \mathrm{~m}$. Colocando las trampas a esta distancia se asume que hay independencia entre las diferentes trampas ya que 1) con esta separación no había interferencia visible (al ojo humano) de la luz producida por cada trampa pitlight y 2) se ha demostrado experimentalmente que la atracción, de diferentes tipos de luz, tiene efecto durante el vuelo a no más de $10 \mathrm{~m}$ ya sea vertical u horizontalmente (Stork et al., 2016). En cada transecto (= tratamiento) seleccionado al azar se colocaron nueve trampas pitfall pasivas o nueve trampas pitlight -con LED de un solo color: azul, blanco o amarillo-, con una separación de 10 m entre cada una (Fig. 2).

La recolecta de coleópteros se realizó durante cinco noches consecutivas del 15 al 19 de abril de 2015. Cabe señalar que el periodo de recolecta coincidió con el de luna nueva, ya que se ha comprobado que las trampas de atracción luminosa son más eficientes en ese periodo, en comparación en el de luna llena (Janzen, 1983; Narváez \& Soriano, 1996). Las trampas se activaron en dos horarios nocturnos de cinco horas, entre las 19:30 a 00:30 horas y de 01:00 a 06:00 horas. Al término de cada periodo de activación, el material recolectado en cada trampa se depositó en frascos debidamente etiquetados.

Los ejemplares de Coleoptera obtenidos fueron conservados en etanol al 70\%, para su posterior contabilización e identificación a nivel de familia y morfoespecie. Los ejemplares permanecen en la Colección Nacional de Insectos (CNIN) y en la Colección Entomológica de la Estación de Biología Tropical Los Tuxtlas, ambas del Instituto de Biología, UNAM.

Análisis de datos. La diversidad alfa de las trampas pitfall y pitlight, para los distintos colores de LED y los dos horarios, se analizó midiendo: 1) la riqueza de especies, mediante curvas de rarefacción, con intervalos de confianza de $95 \%$ y el valor mínimo de abundancia de cada tratamiento como tamaño de muestra estándar; y 2) la diversidad 'verdadera', utilizando el exponencial del índice de Shannon (Jost 2006), de acuerdo con la ecuación:

$$
H_{\alpha}=\exp \left(-\sum_{i=1}^{s} p_{i} \ln p_{i}\right)
$$

Donde $\boldsymbol{s}$ es la riqueza total de especies y $\boldsymbol{p}_{\boldsymbol{i}}$ es la proporción de la especie i en cada tratamiento.

La unidad de medida de la diversidad alfa verdadera es el número de especies efectivas, que puede interpretarse como el número de especies con abundancias iguales, necesarias para alcanzar un determinado valor de un índice de diversidad (Jost, 2006). Las curvas de rarefacción se obtuvieron con el software PAST (Hammer et al., 2001), mientras que la diversidad verdadera con SPADE (Chao \& Shen, 2010).

Dado que también teníamos interés en conocer la eficiencia de la captura en términos de la variación en la composición de especies entre los tratamientos, se analizó el componente beta de la diversidad entre las muestras de coleópteros provenientes de los distintos tipos de trampas y de los dos horarios. Para ello, se utilizó un escalamiento multidimensional no métrico (NMDS, non-metric multidimensional scaling, por sus siglas en inglés), con el que se graficó la disimilitud en la composición de especies entre las muestras, usando como medida de distancia el índice de disimilitud de Bray-Curtis, que considera la abundancia de cada morfoespecie. La diversidad beta también se analizó con el índice de disimilitud de Jaccard, con la finalidad de determinar la variación en la composición de especies entre los distintos tratamientos, sin tener en cuenta la abundancia de las morfoespecies. Los análisis de diversidad beta se realizaron con el software PAST (Hammer et al., 2001).

Con el total de los datos obtenidos por las cinco noches de muestreo se realizó un ANOVA de dos vías, utilizando como factores el tipo de trampa y el horario de captura. Se compararon el número de morfoespecies, el número de familias y la abundancia total de individuos. Posteriormente se utilizó la prueba post hoc de Bonferroni para establecer diferencias significativas entre los dos grupos de datos comparados (trampas pitlight vs. trampas pitfall pasivas). Dicha prueba se ajusta mejor cuando el número de comparaciones es pequeño, a diferencia de la prueba de Tukey, que funciona bien con un número alto de comparaciones. Estos análisis se realizaron mediante el programa SigmaStat 3.1 (Systat software, Inc., Point Richmond, California).

\section{RESULTADOS}

En total se obtuvieron 235 muestras provenientes de los ocho tratamientos implementados (trampas pitfall pasivas y pitlight -con tres colores diferentes- en dos horarios noc- 

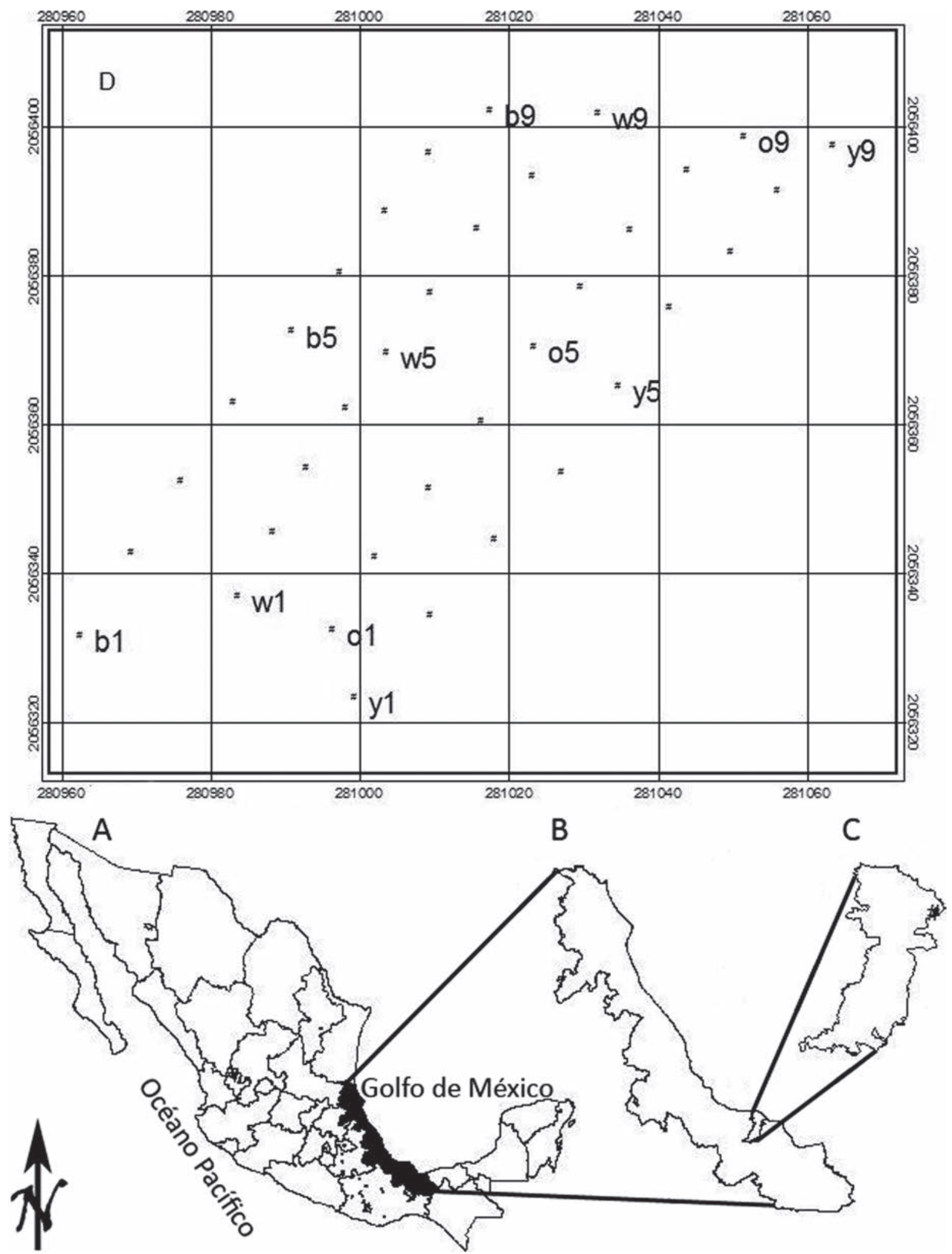

Figura 2. Área de estudio y diseño de los transectos de trampas pitfall y pitlight. Donde A: México; B: Veracruz; C: Santiago Tuxtla. Las trampas se establecieron cada $10 \mathrm{~m}$ en cuatro transectos separados por $10 \mathrm{~m}$ entre sí, como se indica en la parte superior de la imagen: o, trampas pitfall; b, trampas pitlight con LED azules; w, con LED blancos; y c, con LED amarillos. 
turnos), con un esfuerzo total invertido de 135 trampas/ día (número de trampas * número de días de muestreo). De ellas se contabilizaron 1142 ejemplares de Coleoptera pertenecientes a 35 familias y 129 morfoespecies (Cuadro 1). Las familias con un mayor número de especies fueron Chrysomelidae, Curculionidae y Scarabaeidae.

De acuerdo con el tipo de trampa (pitfall pasiva o pitlight) y/o color de LED, 93 (71\%) morfoespecies se obtuvieron en un solo tratamiento, 17 (13\%) en dos y la misma cantidad se capturó en tres tratamientos; sólo cuatro mor- foespecies fueron colectadas con los tres colores de pitlight y con pitfall pasivas. En relación con los horarios, 94 (73\%) morfoespecies fueron exclusivas de alguno de los dos periodos de recolecta nocturna (Cuadro 1).

En las muestras obtenidas con los distintos colores de LED y entre períodos nocturnos de colecta, la abundancia, riqueza y diversidad de familias y morfoespecies fue variable. Entre los tres colores, las trampas con luz azul obtuvieron la mayor abundancia de ejemplares $\left(\mathrm{F}_{(3,30)}=\right.$ 8.22, $p=0.001$; Cuadro 2), mientras que la mayor diversi-

Cuadro 1. Familias y morfoespecies de Coleoptera capturadas con trampas pitlight y pitfall en la Estación de Biología Los Tuxtlas, Veracruz, México. Se muestra el total de ejemplares por familia y por color de LED empleado (S: riqueza de especies; N: total de individuos; Msp: número de morfoespecie; az: azul, bl: blanco, am: amarillo; Ptf: pitfall convencionales. Horarios: 1, de 19:30 a 00:30 horas; 2, 1:00 a 6:00 hrs).

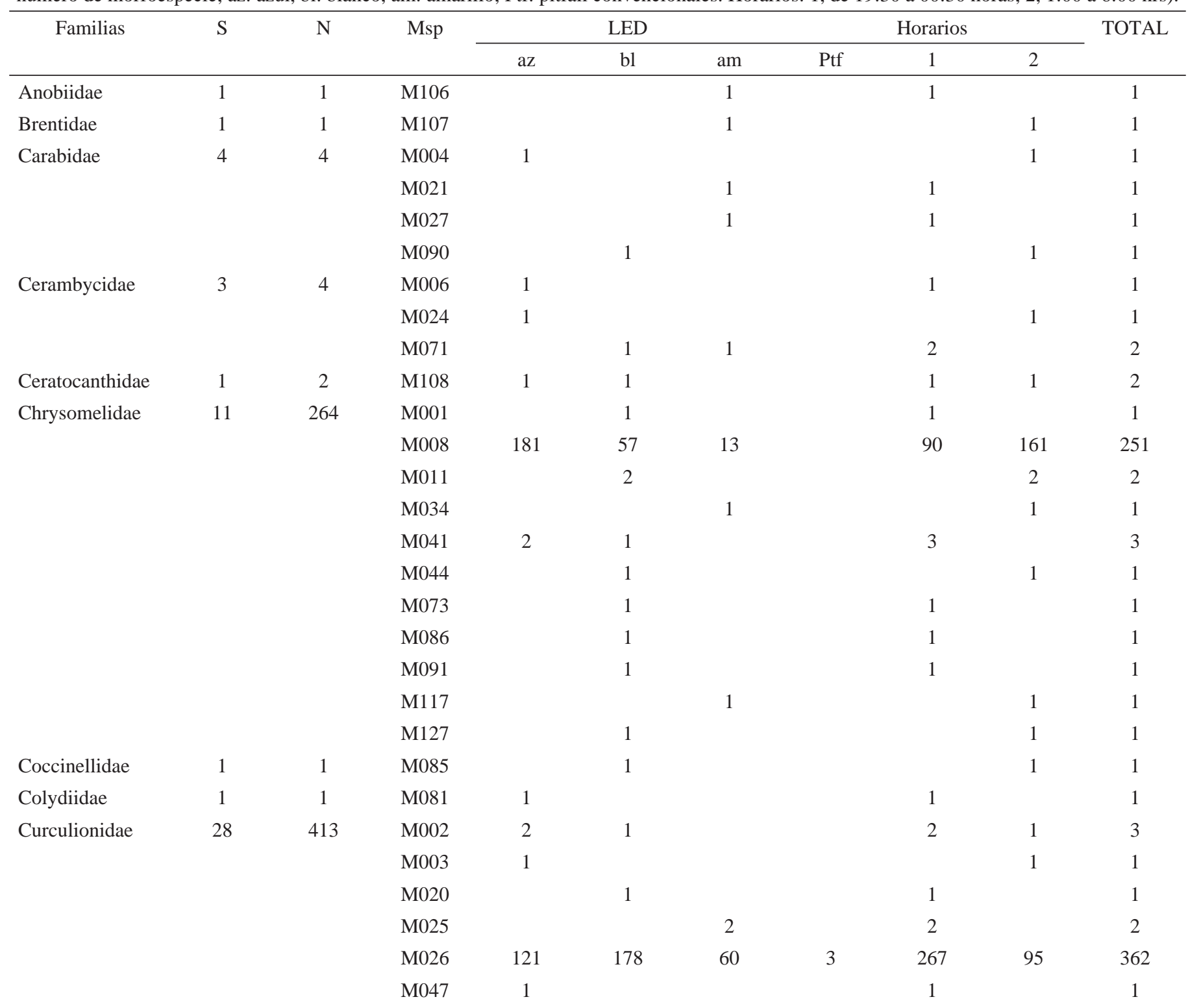


Cuadro 1. Continuación.

\begin{tabular}{|c|c|c|c|c|c|c|c|c|c|c|}
\hline \multirow[t]{2}{*}{ Familias } & \multirow[t]{2}{*}{$\mathrm{S}$} & \multirow[t]{2}{*}{$\mathrm{N}$} & \multirow[t]{2}{*}{ Msp } & \multicolumn{3}{|c|}{ LED } & \multicolumn{3}{|c|}{ Horarios } & \multirow[t]{2}{*}{ TOTAL } \\
\hline & & & & $\mathrm{az}$ & bl & am & Ptf & 1 & 2 & \\
\hline & & & M048 & 1 & & & & 1 & & 1 \\
\hline & & & M050 & 2 & & & & 2 & & 2 \\
\hline & & & M051 & & 1 & & & & 1 & 1 \\
\hline & & & M052 & & & 1 & & 1 & & 1 \\
\hline & & & M053 & 1 & & & & 1 & & 1 \\
\hline & & & M054 & & 1 & & & & 1 & 1 \\
\hline & & & M055 & 6 & 3 & 1 & & 4 & 6 & 10 \\
\hline & & & M056 & 2 & & & & 2 & & 2 \\
\hline & & & M057 & 1 & & & & 1 & & 1 \\
\hline & & & M058 & 1 & 2 & & & 1 & 2 & 3 \\
\hline & & & M059 & & 1 & & & & 1 & 1 \\
\hline & & & M060 & & & 1 & & & 1 & 1 \\
\hline & & & M061 & 2 & 2 & & & 2 & 2 & 4 \\
\hline & & & M062 & & & 1 & & & 1 & 1 \\
\hline & & & M063 & & 1 & & & & 1 & 1 \\
\hline & & & M092 & 1 & & & & 1 & & 1 \\
\hline & & & M116 & & 1 & 1 & & & 2 & 2 \\
\hline & & & M121 & 1 & & & & & 1 & 1 \\
\hline & & & M122 & 2 & 1 & 1 & & 2 & 2 & 4 \\
\hline & & & M123 & & 2 & & & 2 & & 2 \\
\hline & & & M126 & & 1 & & & 1 & & 1 \\
\hline & & & M130 & & & 1 & & & 1 & 1 \\
\hline \multirow[t]{5}{*}{ Elateridae } & 5 & 20 & M018 & 2 & 1 & 9 & & 2 & 10 & 12 \\
\hline & & & M039 & & & 2 & & 1 & 1 & 2 \\
\hline & & & M040 & 1 & & & & 1 & & 1 \\
\hline & & & M046 & & 1 & & & 1 & & 1 \\
\hline & & & M080 & 2 & 1 & 1 & & 3 & 1 & 4 \\
\hline Endomychidae & 1 & 1 & M100 & 1 & & & & & 1 & 1 \\
\hline \multirow[t]{2}{*}{ Erotylidae } & 2 & 2 & M075 & & 1 & & & 1 & & 1 \\
\hline & & & M087 & & 1 & & & & 1 & 1 \\
\hline \multirow[t]{3}{*}{ Lampyridae } & 3 & 43 & M028 & 2 & & 36 & & 37 & 1 & 38 \\
\hline & & & M033 & 1 & 2 & 1 & & 2 & 2 & 4 \\
\hline & & & M037 & & 1 & & & & 1 & 1 \\
\hline Leiodidae & 1 & 1 & M096 & & & 1 & & & 1 & 1 \\
\hline Limnichidae & 1 & 8 & M104 & 2 & 4 & 2 & & 4 & 4 & 8 \\
\hline \multirow[t]{2}{*}{ Lycidae } & 2 & 2 & M036 & 1 & & & & 1 & & 1 \\
\hline & & & M088 & & 1 & & & & 1 & 1 \\
\hline Melandryidae & 1 & 1 & M120 & 1 & & & & & 1 & 1 \\
\hline Meloidae & 1 & 1 & M097 & & 1 & & & & 1 & 1 \\
\hline \multirow[t]{2}{*}{ Mordellidae } & 2 & 2 & M035 & & & 1 & & & 1 & 1 \\
\hline & & & M095 & & 1 & & & & 1 & 1 \\
\hline
\end{tabular}


Cuadro 1. Continuación.

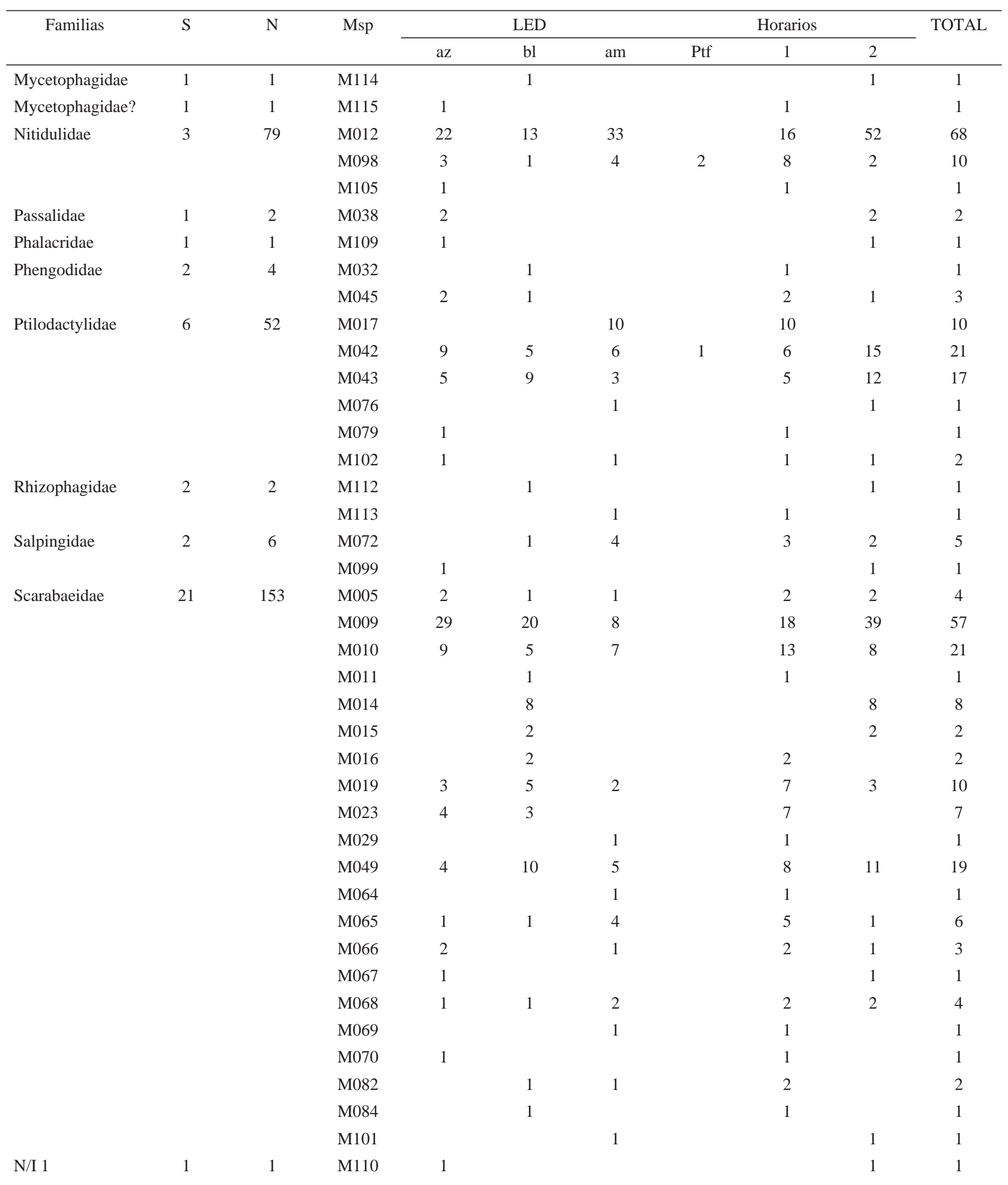


Cuadro 1. Continuación.

\begin{tabular}{|c|c|c|c|c|c|c|c|c|c|c|}
\hline \multirow[t]{2}{*}{ Familias } & \multirow[t]{2}{*}{$\mathrm{S}$} & \multirow[t]{2}{*}{$\mathrm{N}$} & \multirow[t]{2}{*}{ Msp } & \multicolumn{3}{|c|}{ LED } & \multicolumn{3}{|c|}{ Horarios } & \multirow[t]{2}{*}{ TOTAL } \\
\hline & & & & $\mathrm{az}$ & $\mathrm{bl}$ & $\mathrm{am}$ & Ptf & 1 & 2 & \\
\hline N/I 2 & 1 & 2 & M007 & 1 & & 1 & & 1 & 1 & 2 \\
\hline \multirow[t]{7}{*}{ Staphylinidae } & 2 & 35 & M093 & 12 & 14 & 4 & 1 & 24 & 7 & 31 \\
\hline & & & M129 & 4 & & & & 3 & 1 & 4 \\
\hline & & & M030 & & & 2 & & & 2 & 2 \\
\hline & & & M103 & & 1 & & & 1 & & 1 \\
\hline & & & M124 & & 1 & & & 1 & & 1 \\
\hline & & & M128 & & & & 1 & & 1 & 1 \\
\hline & & & M074 & & 2 & & & 2 & & 2 \\
\hline \multirow{5}{*}{ Tenebrionidae } & & & M078 & & 1 & & & 1 & & 1 \\
\hline & & & M083 & & & 2 & & 1 & 1 & 2 \\
\hline & & & M089 & 1 & & & & & 1 & 1 \\
\hline & & & M118 & & 1 & & & & 1 & 1 \\
\hline & & & M119 & & & 1 & & 1 & & 1 \\
\hline Total general & 129 & 1142 & - & 473 & 400 & 261 & 8 & 623 & 519 & 1142 \\
\hline
\end{tabular}

dad se logró con las trampas de LED amarillos y la menor con los azules (20.02 y 10.13 especies efectivas, respectivamente; Cuadro 2). Entre horarios de muestreo, la mayor diversidad se obtuvo durante el segundo periodo nocturno (15.25 especies efectivas) (Cuadro 2). La curva de acumulación de especies mostró que en las trampas con LED blancos resultaron las más ricas en especies (Fig. 3a); así mismo, durante el primer periodo nocturno se obtuvo una mayor riqueza de morfoespecies (Fig. 3b). Las trampas pitlight de un mismo color LED también mostraron diferencias en la riqueza obtenida en cada horario de activación (Fig. 4).

El análisis NMDS mostró una similitud mayor entre las muestras de las trampas pitlight azules y blancas del horario 1 , mientras que el resto de las muestras fueron muy disímiles entre sí (Fig. 5). El índice de Jaccard permitió identificar con mayor detalle las diferencias en la disimilitud total en la composición de especies entre colores de LED y entre horarios, que fueron siempre mayores a 70\% (Cuadro 3). Lo mismo se observó al comparar las recolectas obtenidas en los distintos horarios, ya fuera con los datos totales o separados por tipo de luz (Cuadro 4).
$\mathrm{Al}$ analizar la riqueza de morfoespecies, el ANOVA mostró diferencias significativas entre tipos de trampas $\left(\mathrm{F}_{(3,32)}=17.38, \mathrm{p}<0.001\right)$, pero no entre los dos horarios $\left(F_{(1,32)}=0.24, p=0.62\right)$, ni en la interacción entre el horario y el tipo de trampa $\left(\mathrm{F}_{(3,32)}=0.68 \mathrm{p}=0.56\right)$. Las principales diferencias se encontraron en la riqueza de especies obtenida con las trampas pitfall pasivas respecto a las pitlight con LED blancos $(\mathrm{p}<0.001)$, azules $(\mathrm{p}<0.001)$ o amarillos $(p=0.001)$, pero no hubo diferencias significativas entre estas últimas ( $>0.05)$. Dentro de los dos horarios también se encontraron diferencias significativas de acuerdo con el tipo de trampa entre las pitfall pasivas en relación con las pitlight con LED blancos, azules o amarillos $(\mathrm{p}<0.001$; Fig. 6a).

La riqueza de familias de coleópteros entre tipos de trampas fue significativamente diferente $\left(\mathrm{F}_{(3,32)}=23.44 \mathrm{p}=\right.$ $0.0001)$; no así entre horarios $\left(\mathrm{F}_{(1,32)}=0.00 \mathrm{p}=1.00\right)$, ni en la interacción de éstos y el tipo de trampa $\left(\mathrm{F}_{(3,32)}=0.30\right.$ $\mathrm{p}=0.82$ ). También hubo diferencias significativas entre trampas pitfall respecto a las pitlight con LED blancos $(p<0.001)$, con LED azules $(p<0.001)$ o con LED amarillos $(p<0.001)$. Entre trampas pitlight, aquellas con luz azul y blanca tuvieron el mismo número de familias, mientras 
Cuadro 2. Diversidad de Coleoptera (Insecta) obtenida con trampas pitfall pasivas (sin LED) y con trampas pitlight con LED de distintos colores en la Estación de Biología Los Tuxtlas, Veracruz, México.

\begin{tabular}{lccccc}
\hline \multicolumn{1}{c}{$\begin{array}{c}\text { Horario/Color } \\
\text { de LED }\end{array}$} & Número de familias & Abundancia & \multicolumn{2}{c}{ Riqueza de morfoespecies } & $\begin{array}{c}\text { Diversidad } \\
\text { (exp H') }\end{array}$ \\
\cline { 4 - 5 } & & & Total & Exclusivas & \\
Horario 1 & & & & & 13.24 \\
(19:30 a 00:30 hr) & 25 & 623 & 83 & & 15.27 \\
Horario 2 & & & & 45 & 10.13 \\
(01:00 a 06:00 hr) & 31 & 519 & 79 & 29 & 12.23 \\
Azul & 24 & 473 & 61 & 37 & 20.02 \\
Blanco & 23 & 400 & 69 & 27 & 4.45 \\
Amarillo & 20 & 261 & 55 & 1 & $\mathbf{1 6 . 8 5}$ \\
Sin LED & 4 & 8 & 5 & $\mathbf{9 4}$ & \\
Total & $\mathbf{3 7}$ & $\mathbf{1 1 4 2}$ & $\mathbf{1 2 8}$ & & \\
\hline
\end{tabular}
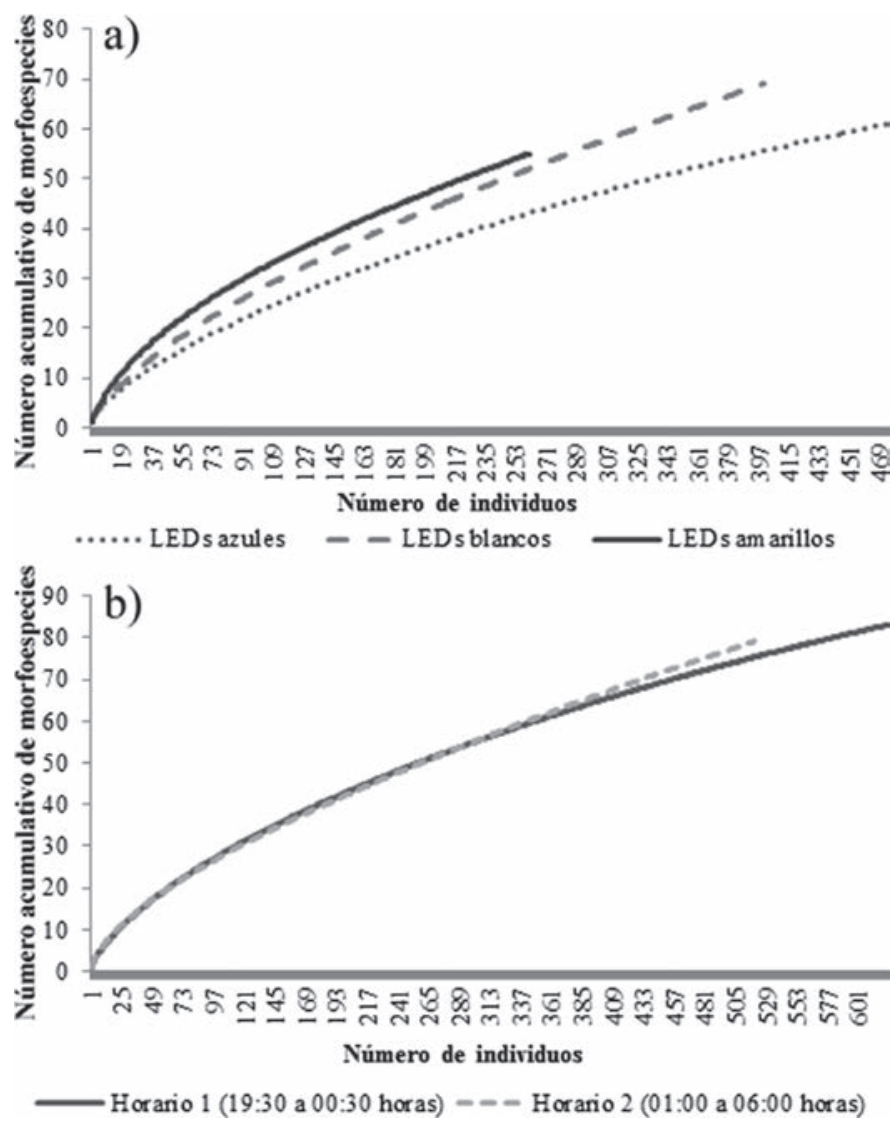

Figura 3. a) Comparación de la riqueza de especies de coleópteros de tres trampas pitlight con LED azules (línea punteada), LED blancos (guiones) y LED amarillos (línea sólida); b) Riqueza de especies de coleópteros recolectados en dos periodos nocturnos de activación de trampas pitlight con LED (Horarios: 1, de 19:30 a 00:30 horas, línea oscura; 2, 01:00 a 06:00 horas, línea punteada). los LED amarillos capturaron apenas tres familias menos. Dentro de los horarios, se encontraron diferencias significativas entre las trampas pitfall en relación con las pitlight con LED blancos, azules y amarillos (Fig. 6b).

La abundancia total de Coleoptera obtenida con trampas pitlight fue muy superior a aquella alcanzada con pitfall: el 99.5\% fue recolectado con las primeras. El ANOVA mostró que esas diferencias son significativas $\left(\mathrm{F}_{(3,32)}=14.10 \mathrm{p}<0.001\right)$, al igual que las diferencias entre las trampas pitfall y las pitlight con LED blancos $(\mathrm{p}<0.001)$, azules $(\mathrm{p}<0.001)$ o amarillos $(\mathrm{p}=0.015)$. Entre horarios, las diferencias no fueron significativas $\left(\mathrm{F}_{(1,32)}=\right.$ $0.90 \mathrm{p}=0.34)$, ni en la interacción horario-tipo de trampa $\left(\mathrm{F}_{(3,32)}=1.81 \mathrm{p}=0.16\right)$. Dentro del horario 1 las trampas pitfall sólo fueron significativamente distintas a las trampas con LED blancos y azules, en tanto que en el horario 2 sólo se encontraron diferencias entre las trampas pitfall y las trampas con LED azules (Fig. 6c). Entre trampas pitlight, sólo se encontraron diferencias significativas entre ambos horarios en las trampas con LED blancos (Fig. 6c).

\section{DISCUSIÓN}

Debido a que las capturas con trampas pitfall pasivas son esencialmente incidentales y su eficiencia incrementa con la adición de atrayentes, la mayoría de los estudios que las emplean están dirigidos a grupos muy particulares de insectos que responden a un cebo específico, como los coprófagos (e. g. Scarabaeidae, Halffter, 2006; Montes de Oca, 2001), y no a grupos de niveles taxonómicos mayores. 


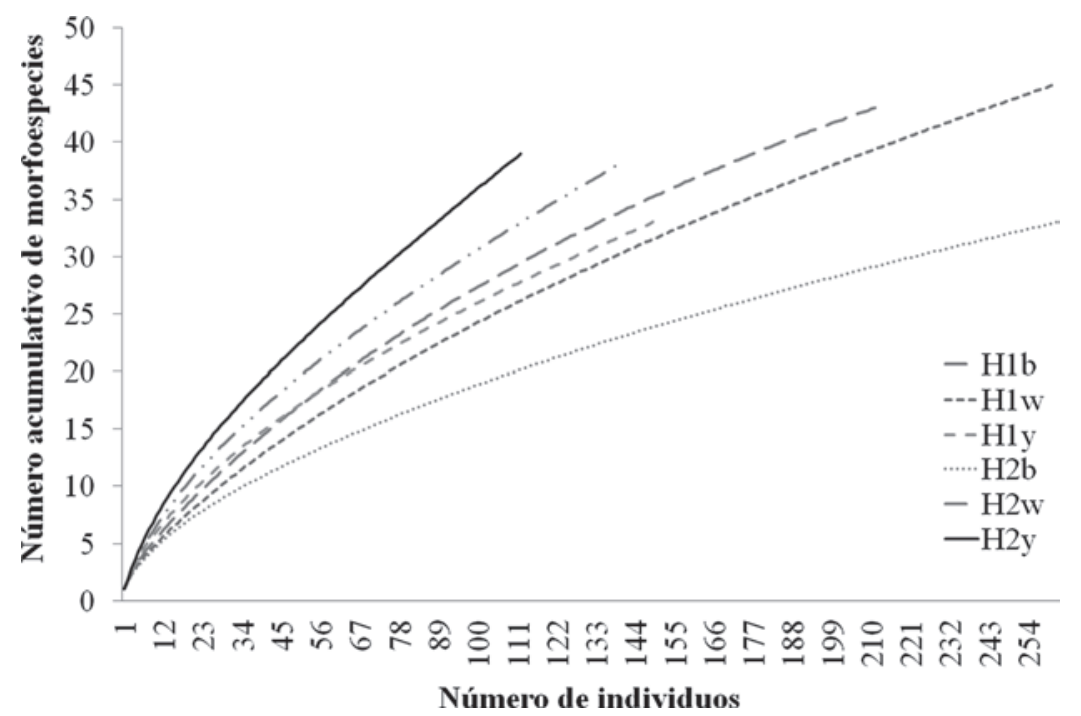

Figura 4. Comparación de la riqueza de especies de coleópteros obtenidas en dos periodos de muestreo nocturno (H1: de 19:30 a 00:30 horas; y H2: 01:00 a 06:00 horas) con tres tipos de trampas pitlight con LED (b: azules; w: blancos; y: amarillos).

En este estudio se observó que la adición de una fuente luminosa en trampas pitfall mejora notablemente la recolecta: la efectividad de las trampas pitlight en la captura de coleópteros es mucho mayor que la de trampas pitfall pasivas; aunque dicha efectividad no depende tanto de la

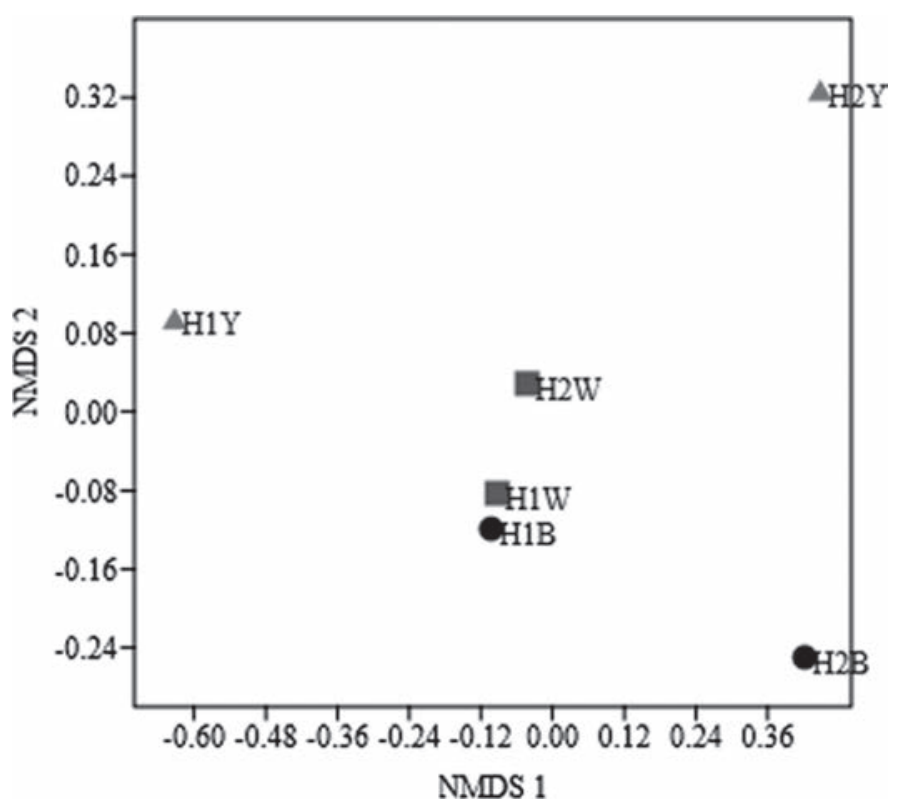

Figura 5. Escalamiento multidimensional no métrico (NMDS) basado en la composición y abundancia de especies de las recolectas de coleópteros obtenidas con trampas pitlight con LED de tres distintos colores (B: azul, W: blanco, Y: amarillo) durante dos períodos nocturnos de activación (H1 y H2). Valor de stress=0. longitud de onda, sino del horario de muestreo. Además, las trampas pitlight atrajeron una cantidad considerable de familias y morfoespecies en un periodo corto de tiempo y con un esfuerzo mínimo de los colectores. Dado su tamaño y portabilidad este tipo de trampas son eficientes para el establecimiento de monitoreos a largo plazo de diferentes grupos de insectos.

En comparación con otros trabajos llevados a cabo en ecosistemas tan diversos como las selvas tropicales, nuestros resultados obtenidos con trampas pitlight son superiores; por ejemplo, Teixeira y colaboradores (2009) encontraron 24 familias de coleópteros en un muestreo anual con trampas pitfall pasivas, mientras que aquí se capturaron 35 familias en un muestreo de tan sólo cinco días. Nuestros hallazgos apoyan la idea de que el uso de trampas pitlight representa un método muy eficiente para inventarios rápidos de la biodiversidad de este orden.

Por otro lado, las familias más abundantes y ricas en especies que se registraron en este estudio (Chrysomelidae, Curculionidae y Scarabaeidae), también suelen ser muy diversas en muestreos con trampas de atracción luminosa o pitfall convencionales. Sin embargo, es importante destacar que los miembros de otras familias consideradas raras o que usualmente no son capturadas con trampas de atracción luminosa, fueron capturadas por las trampas pitlight, como es el caso de Lampyridae y Endomychidae (Cuadro 1).

Resulta interesante que la mayoría de morfoespecies (alrededor de 70\%) fueron capturadas exclusivamente en 
Cuadro 3. Disimilitud en la composición de especies de Coleoptera entre los diferentes tipos de luz utilizados en las trampas pitlight y entre los dos horarios de muestreo: 1) 19:30 a 00:30 horas y 2) 01:00 a 06:00 horas, de acuerdo con el índice de disimilitud de Jaccard.

\begin{tabular}{llccc}
\hline $\begin{array}{c}\text { Horario/Tipo } \\
\text { de luz }\end{array}$ & & Horario 2 & Azul & Blanca \\
\hline Horario 1 & $\beta \mathrm{cc}$ & 0.73 & & \\
Blanca & $\beta \mathrm{cc}$ & & 0.71 & \\
Amarilla & $\beta \mathrm{cc}$ & & 0.73 & 0.75 \\
\hline
\end{tabular}

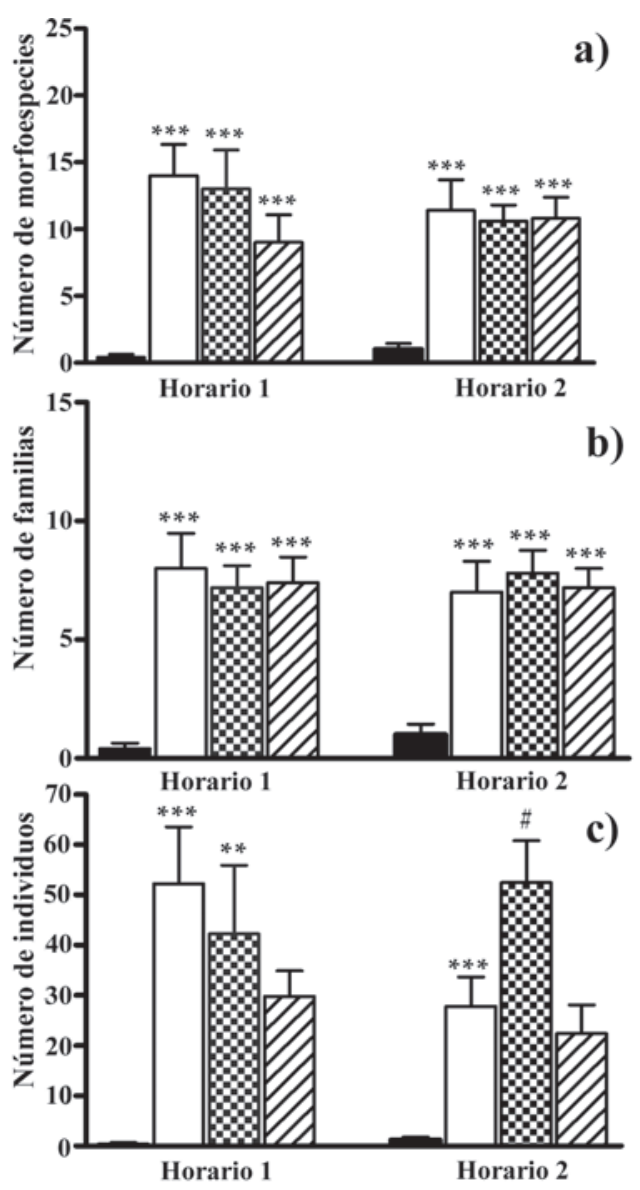

-Pitfall $\square$ Pitlight blanca $\$$ Pitlight azul $\square$ Pitlight amarilla

Figura 6. Diferencias entre distintos tipos de trampa en dos horarios de muestreo, de acuerdo con: a) número de morfoespecies de coleópteros (Bonferroni: ${ }^{* * *} \mathrm{p}<0.001$ vs. pitfall); b) número de familias de Coleoptera (Bonferroni: $* * * \mathrm{p}<0.001$ vs. pitfall); y c) número de individuos (Bonferroni ${ }^{* * *} \mathrm{p}<0.001,{ }^{* *} \mathrm{p}<0.01$ vs. pitfall; $\# \mathrm{p}<0.05$ vs. horario 1).

un solo color de LED, lo que podría sugerir una preferencia de los coleópteros por un color de luz particular. No obstante, también es necesario mencionar que un conjunto considerable de las morfoespecies registradas (71\%), está representado únicamente por uno o dos ejemplares, por lo que la preferencia de los coleópteros por un color particular de LED no se pudo evaluar con precisión.

Contrario al supuesto de que los insectos son más atraídos por la luz visible con longitudes de onda más cortas (Ashfaq et al., 2005), en este estudio no se observaron diferencias significativas entre la riqueza y la abundancia obtenida por uno u otro color de LED, tampoco por aquellos de longitudes de onda más cortas, que en este caso corresponden a los de color azul; es decir, que no hay diferencias notables en el número de especies y la cantidad de cada especie capturados con los distintos colores de LED. Sin embargo, aunque no se encontraron dichas diferencias esperadas, los análisis de la disimilitud en la composición de especies (diversidad beta), mostraron la existencia de un alto recambio de especies entre trampas pitlight de tres colores distintos ( $>70 \%$ ), como resultado de la alta exclusividad de las especies a uno u otro tipo de luz mencionada en el párrafo anterior. Por esa razón, la combinación de trampas pitlight de distintos colores de LED podría ser más eficiente para el muestreo de coleópteros, en comparación con el empleo de luz de un solo color. La distancia mínima entre trampas es también importante ya que si esta no es la adecuada puede existir interferencia entre ellas. Esta interferencia depende del tipo de luz, pero también el estrato que se esté muestreando. Stork y colaboradores (2016) reportaron que verticalmente 10 metros es la distancia mínima que puede haber, ya que muchos insectos pueden ser atraídos hasta casi $17 \mathrm{~m}$ hacia una fuente de luz. Sin embargo, de manera horizontal se ha reportado que diversas especies de insectos, entre estas varias de Coleoptera, son atraídos en vuelo a menos de 5 metros de la fuente de luz, aunque hay algunas polillas que pueden ser atraídas hasta $80 \mathrm{~m}$ de distancia (Stork et al., 2016; Truxa \& Fiedler, 2012).

Otra de las interrogantes de este trabajo está relacionada con la cantidad de horas necesarias para obtener una muestra representativa de la fauna de coleópteros nocturnos y con los horarios más adecuados para realizar el muestreo. Los resultados obtenidos en los dos periodos en que se dividieron las noches de muestreo, muestran que la riqueza y abundancia de morfoespecies sólo fue ligeramente mayor en el primer periodo, sin diferencias estadísticamente significativas entre ambos periodos. Sin embargo, es importante recalcar que los valores de las diferencias en la composición de especies entre ambos periodos de capturas fueron mayores a 70\% y la cantidad de especies exclusivas de cada horario también fue alto. Esto significa que el recambio en la composición de morfoes- 
Cuadro 4. Disimilitud en la composición de especies de Coleoptera capturadas con trampas pitlight de tres colores de LED durante dos períodos nocturnos de recolecta, obtenida con el índice de disimilitud de Jaccard.

\begin{tabular}{|c|c|c|c|c|c|c|}
\hline \multicolumn{2}{|c|}{ Horario - tipo de luz } & \multicolumn{2}{|c|}{ Horario 1} & \multicolumn{3}{|c|}{ Horario 2} \\
\hline & & Azul & Blanca & Amarilla & Azul & Blanca \\
\hline Horario 1 (19:30 a 00:30 hrs.) & Blanca & 0.71 & & & & \\
\hline \multirow[t]{2}{*}{ Horario 2 (01:00 a 06:00 hrs.) } & Azul & 0.75 & 0.70 & 0.75 & & \\
\hline & Blanca & 0.77 & 0.80 & 0.84 & 0.78 & \\
\hline
\end{tabular}

pecies de coleópteros varía considerablemente durante la noche, por lo que un muestreo nocturno más prolongado sería mucho más eficiente (de crepúsculo a crepúsculo).

Este estudio muestra que la adición de LED en las trampas de caída (pitlight) representa una modificación novedosa en los métodos de captura de coleópteros nocturnos que, aparentemente, resulta más eficiente en términos de costo, portabilidad, esfuerzo de muestreo y diversidad obtenida, en comparación con trampas pitfall pasivas (e.g. Teixeira et al., 2009), trampas de atracción luminosa comunes y con otros modelos de pitlight (e.g. Hébert et al., 2000), por lo que se recomienda explorar más el uso de este tipo de trampas, para comparar su utilidad y efectividad frente a distintos tipos de recolecta y por períodos más largos de tiempo. Aunque con nuestros resultados, podemos por lo menos recomendar su implementación en estudios enfocados al análisis de la biodiversidad y la conservación, los cuales generalmente buscan obtener una muestra representativa en periodos cortos de tiempo.

AGRADECIMIENTOS. Al Posgrado en Ciencias Biológicas de la UNAM por el apoyo para la estancia en la Estación de Biología Los Tuxtlas a través del curso "Métodos de Muestreo y Análisis en Comunidades”. Al Dr. Santiago Zaragoza Caballero y D.G. Rafael Perdomo Manjarrez, por el rediseño y construcción de las trampas pitlight. CXPH quiere agradecer especialmente a Rosamond Coates Estrada, Directora de la Estación de Biología Los Tuxtlas y Marta Madora Astudillo, encargada de la Colección de Entomología de la misma Estación, por el apoyo brindado en sus instalaciones.

\section{LITERATURA CITADA}

Alcázar-Ruiz, J. A., Morón-Ríos, A. \& Morón, M. A. (2003). Fauna de Coleoptera Melolonthidae de Villa Las Rosas, Chiapas, México. Acta Zoológica Mexicana (n.s.), 88, 59-86.

Alexander, K. N. A. (2014). A review of the scarce and threatened beetles of Great Britain, Species Status No. 16. Natural England Commissioned Report NECR134, United Kingdom.

Alonso, L. E., Deichman, J. L. Mckenna, S. A., Naskcreicki, P. \&
Richards, S. J. (2011). Still counting...: Biodiversity Exploration for Conservation: The first 20 years of the Rapid Assessment Program. Arlington, Virginia, United States of America: Conservation International.

Arnett, R. H. Jr., Thomas, M. C., Skelley, P. E. \& Frank, J. H. (2002). American Beetles. Vol. 2. Polyphaga: Scarabaeoidea through Curculionoidea. CRC Press, Boca Raton, Florida.

Ashfaq, M., Khan, R., Khan, M., Rasheed, F. \& Hafeez, S. (2005). Insect orientation to various color lights in the agricultural biomes of Faisalabad. Pakistan Entomologist, 27, 49-52.

Biocom, (2014). LUMInous miniature trap for NOCturnal insects, LUMINOC C. http://web.archive.org/web/20050129051014/ http://biocom.ca/ (Accesed on April 2017).

Burkett, D., Butler, J. \& Kline, D. (1998). Field evaluation of colored light-emitting diodes as attractants for woodland mosquitoes and other Diptera in North Central Florida. Journal of the American Mosquito Control Association, 14, 186-195.

Chagnon, M., Hébert C. \& Pare, D. (2000). Community structures of Collembola in sugar maple forests: relations to humus type and seasonal trends. Pedobiologia, 44, 148-174.

Chao, A. \& Shen, T. J. (2010). Programa SPADE (Species Prediction and Diversity Estimation). http://chao.stat.nthu.edu.tw/blog/software-download/spade/ (Accesed on April 2015).

Cohnstaedt, L., Gillen J. \& Munstermann, L. (2008). Light-emitting diode technology improves insect trapping. Journal of the American Mosquito Control Association, 24, 331-334.

Conanp (Comisión Nacional de Áreas Naturales Protegidas). (2006). Programa de Conservación y Manejo Reserva de la Biosfera Los Tuxtlas. Dirección General de Manejo para la Conservación y la Dirección Regional Centro y Golfo, CONANP, México.

Cutz-Pool, L. \& Virginia, V. (2013). Arañas (Arachnida: Araneae) de la reserva "Los Huiros” del ejido Tres Garantías, Quintana Roo, capturadas mediante dos técnicas de recolecta, pp. 89-94. In: A. Equihua-Martínez, E. G. Estrada-Venegas, J. A. Acuña-Soto \& M. P. Chaires-Grijalva (eds). Entomología mexicana vol. 2. Sociedad Mexicana de Entomología, A.C. y Colegio de Postgraduados, Montecillo, Texcoco, Estado de México, México.

Drenen, T., Haitz, R. \& Tsao, J. (2000). Transforming the lighting sector with semiconductor lighting technologies. Sandia National Laboratories, 34, 67-71.

Fagundes, C. K., Di Mare, R. A., Wink, C. \& Manfio, D. (2011). Diversity of the families of Coleoptera captured with pitfall traps in five different environments in Santa Maria, RS, Brazil. Brazilian Journal of Biology, 71, 381-390. 
Halffter S., G. (2006). Análisis de las relaciones entre las diversidades alfa, beta y gamma a distintos niveles de escala espacial: procesos históricos y ecológicos que intervienen. IV Etapa. Informe final Sistema Nacional de Información sobre la Biodiversidad-Comisión Nacional para el Conocimiento y Uso de la Biodiversidad (SNIB-CONABIO) proyecto No. BE012. Instituto de Ecología A. C. México, D. F.

Hammer, Ø., Harper, D. A. T. \& Ryan, P. D. (2001). PAST: Paleontological Statistics Software Package for Education and Data Analysis. Palaeontologia Electronica, 4, 1- 9. http://palaeo-electronica.org/2001_1/past/issue1_01.htm (Accesed on April 2015).

Hansen, J. E. \& New, T.H. (2005). Use of barrier pitfall traps to enhance inventory surveys of epigaeic Coleoptera. Journal of Insect Conservation, 9, 131-136.

Heap, M. (1988). The pit-light, a new trap for soil-dwelling insects. Journal of the Australian Entomological Society, 27, 239-240.

Hébert, C., Jobin, L., Frechette M. \& Pelletier, G. (2000). An efficient pit-light trap to study of beetle diversity. Journal of Insect Conservation, 4, 191-202.

Holopainen, J. K. (1992). Catch and sex ratio Carabidae (Coleoptera) in pitfall traps filled with ethylene glicol or water. Pedobiologia, 36, 257-261.

Jost, L. (2006). Partitioning diversity into independent alpha and beta components. Ecology, 88, 2427-2439.

Méndez-Aguilar, M. J., Castro-Ramírez, A. E., Alvarado-Barrantes, R., Pacheco-Flores, C. \& Ramírez-Salinas, C. (2005). Eficacia de dos tipos de recolecta para registrar la diversidad de melolóntidos nocturnos (Coleoptera: Scarabaeoidea). Acta Zoológica Mexicana (n.s.) 21, 109-124.

Montes de Oca, E. (2001). Escarabajos coprófagos de un escenario ganadero típico de la región de Los Tuxtlas, Veracruz, México: importancia del paisaje en la composición de un gremio funcional. Acta Zoológica Mexicana (n.s.), 82, 111-132.

Price, B. \& Baker, E. (2016). NightLife: A cheap, robust, LED based light trap for collecting aquatic insects in remote areas. Biodiversity Data Journal 4: e7648.

Racey, P. A. \& Swift, S. M. (1985). Feeding ecology of Pipistrellus pipistrellus (Chiroptera: Vespertilionidae) during pregnancy and lactation. I, Foraging behaviour. Journal of Animal Ecology, 54, 205-215.

Scalercio, S., Infusino, M. \& Woiwod, I. P. (2009). Optimizing the sampling window for moth indicator communities. Journal of Insect Conservation, 13, 583-591.

Stork, N. E., Stone, M. \& Sam, L. (2016). Vertical stratification of beetles in tropical rainforests as sampled by light traps in North Queensland, Australia. Austral Ecology, 41, 168-178.

Teixeira, C. C. L., Hoffmann, M. \& Silva-Filho, G. (2009). Community of soil fauna Coleoptera in the remnants of lowland Atlantic Forest in state of Rio de Janeiro, Brazil. Biota Neotropica, 9, 9195.

Therrien, F., Chagnon, M. \& Hébert, C. (1999). Biodiversity of Collembola in sugar maple (Aceraceae) forests. The Canadian Entomologist, 13, 613-628.

Woodcock, B. A. (2014). Pitfall trapping in ecological studies. Pp. 37-57. In: S. Leather, J. H. Lawton \& G. E. Likens (eds). Insect sampling in forest ecosystems. Blackwell Publishing, Australia.

Zhang, Z. Q. (2013). Phylum Arthropoda. In Animal Biodiversity: An outline of higher-level classification and survey of taxonomic richness (Adenda 2013). Zootaxa 3703, 1-82. 